\title{
Biomedical Adaptive Educational Hypermedia System: a Theoretical Model for Adaptive Navigation Support
}

\author{
Maria Aparecida Fernandes Almeida and Fernando Mendes de Azevedo \\ Federal University of Santa Catarina, \\ Brazil
}

\section{Introduction}

The first Adaptive Hypermedia System (AHS) was presented in the early nineties (Furuta \& Scotts, 1990), (Ginderen, 1990). However, this model was not based in World Wide Web (WWW). The first use in WWW appeared in 1996 (Brusilovsky, 1996). Currently, AHS are used for information recovery; interface adaptation, user assistance, learning support, dialogue, cooperation support and others applications. The AHS have become an alternative to traditional static hypermedia systems with the incorporation of dynamics in Web applications that need to adapt according users.

Brusilovsky (1996) defines an AHS as hypertext and hypermedia system that reflect some user characteristic in a model and can adapt several visible aspects to the system for final user. In other words, the system should satisfy three main criteria: it should be hypertext or hypermedia, it should have an user model and it should be able to adapt using the user model.

Brad Campbell and Joseph Goodman (1987) developed a model known as Hypertext Abstract Machine (HAM). The Dexter Hypertext Reference Model was originated from NIST Hypertext Standardization Workshop (Halasz \& Schwartz, 1994). These models served as a formal reference and informal to the abstractions found in a wide range of hypertexts systems. The Trellis Model, developed by Stotts and Furuta (1990) was based on three elements: information content, navigation structures and application dynamics control. In 1992, the Tower Model was presented in the Workshop of National Institute of Standard and Technology. It was an object-oriented model, based on HAM model (Campbell \& Goodman, 1987). The Amsterdam Hypermedia Model (AHM) developed by Hardman (1994) was based on Dexter Model. This model introduced the concept of time necessary to the complex relationships in hypermedia modelling. The AHM Model included temporal and space relationships among the elements and paid attention in behavior definition of links among the dynamic media groups.

The work of Pagano (1992) presented a theoretical definition of hypertext. This model was extended by Almeida (Almeida, 2002) for educational hypermedia applications. The hypertexts and hypermedia systems that were mentioned previously are not adaptive. One the first efforts towards to put adaptivity of hypertexts / hypermedia systems was due to Benyon (1993) that proposed a model of AHS with modules composed User Model, Domain 
Model and Interaction Model. Brusilovsky (1996) also proposed a model that contained User Model warehoused in a User Model Base and Adaptive Interface. The Adaptive Hypermedia Application Model (AHAM), proposed by DeBra (1993) it is a variant from Dexter Model, including the teaching model composed by pedagogic rules that are used by an adaptive engine to generate the features specifications. The AHAM uses the relationship concept among the components. The Adaptive Hypermedia Architecture (AHA), also developed by DeBra (1993) was considered as an AHS architecture but it contains an authorship tool that uses client-server technology. Today, the growth of distance education has led to the growth of Adaptive Educational Hypermedia Systems (AEHS).

The Web-based Education led to the development of Adaptive Educational Hypermedia Systems (AEHS). The AEHS are highly configurable systems that necessarily involve the user modelling. AEHS must to represent and to support the dynamic environment and user interaction. The AEHS become complex systems with many mechanisms of adaptation and several ways to presentation the interface. In these systems must be guaranteed a proper construction and that the system has a proper behaviour.

Many adaptive hypermedia systems were developed without use of modeling techniques; the developers have not followed the implementation methodology. Due to the countless applications of the AHS and the hypermedia technology development its is necessary to represent arbitrary references and mechanisms combination for specification these systems. A model is a theoretic referential to formalizes all the characteristics and essential functions that can be included in any hypertext application. The model should represent the static and dynamics structure of hypertext system. On Reference Models (Halasz \& Schwartz, 1994) the conceptual abstracts of hypertext / hypermedia systems were created to establish standards to interchange different hyperdocuments among systems. The Design Method models (Rossi, 2010) brought a solid and systematic set of phases that helps the development of hypermedia systems. The hypermedia systems can be built obeying the phases of the development process: analysis, project, implementation and maintenance.

The growing AEHS complexity, whose operation is highly dependent of the users behaviors and of the own system, it turned a construction need of reliable systems whose ambiguities can be reduced by formal specifications in development process. In the AEHS specification, it is necessary to consider the state transitions, the functional behavior, the time relationships between the components and the multiple media integration to effectiveness from its usage.

This work presents a formal model of AEHS in the Biomedical Engineering based on the Category Theory (CT) (Arbib, 1975), (Adamek, 2004) in way to contribute with the development of these systems. The categorical approach in the Adaptive Educational Hypermedia System on Medical Education was proposed by Almeida and Azevedo (2008). The formal model was denominated of Biomedical Adaptive Educational Hypermedia System (B-AEHS). The components of an AEHS were modelled as objects and sub-objects of categories. The system parts were treated as categorical objects and their common aspects were explored to generate universal properties.

The CT is known as the "theory of structure" and has been applied to deal with the formalization of computer systems (Adamek, 2004), (Awodey, 2006), (Barrett \& Mackaay, 2006). The categorical principles have been used to formalize different mathematical models of behaviour of systems, its specifications and its logical outputs (Fiadeiro, 2005).

The categorization of AEHS can be defined in several levels, in different structures. The categorical language simplifies the abstraction facilitating the uniform conception of these 
systems. The CT is a formal method useful in the definition of objects that have a universal property because it reveals how structures of different characteristics are related. The notion of abstraction is essential in the application of a formal method. The first step is to produce an abstract specification that characterizes the essential properties of the problem, to declare what is necessary to describe the problem and how this can be achieved (Gunawardena, 1996).

At some level of generalization an AEHS consists of a set of nodes or hyper documents connected by links. Each node contains some local information and links related to other nodes. The AEHS may also include an index or map with links to all available nodes. In this situation, the adjustment may occur at the level of content of the nodes or at the level of links, indexes and maps.

The adaptivity in AEHS is the ability to change dynamically the system according to the needs of users. All student interaction with the system is made by the adaptive interface. The adaptive interface is built from information about the user. There are two distinct areas of adaptation: adaptive presentation (content level adaptation) and adaptive navigation support (link level adaptation) (Brusilovsky, 2001). Adaptive presentation is concerned with the adaptations of text and multimedia. Adaptive navigation support is related into direct guidance, link hiding, sorting, annotation and hypermedia map adaptation. The adaptive navigation techniques are used to handle links and nodes for adapt the dynamic navigation features according to the state of the user model (Brusilovsky, 2002).

The chapter was structured as follows. In the next Section we present the basic concepts of the Category Theory. In Section 3 we present the formal method for the description of the structure of the adaptive navigation in the B-AEHS. In Section 4 we present a categorical model of an educational support system in Neuroanatomy. In a concluding Section 5, we give some final remarks.

\section{Category theory}

The CT (Arbib, 1975) was introduced as programs specification language in end of sixties. The categories can be:

- Real: are categories that exist in real world and can be represented by abstract categories.

- Abstract: are mathematical entities that can have several interpretations.

To characterize an abstract category it is necessary to identify the objects and morphism.

Definition 1. A category C consists of the following data (Adamek, 2004):

- Objects: $\mathrm{Ob}_{1}, \mathrm{Ob}_{2}, \mathrm{Ob}_{3}, \ldots$

- Arrows, called morphisms: $f, g, h, \ldots$

- For each arrow $f$ there are given objects:

$$
\operatorname{dom}(f), \operatorname{cod}(f)
$$

These objects are called the domain and codomain of $f$. We write:

$$
f: \mathrm{Ob}_{1} \rightarrow \mathrm{Ob}_{2}
$$

to indicate that $\mathrm{Ob}_{1}=\operatorname{dom}(\mathrm{f})$ and $\mathrm{Ob}_{2}=\operatorname{cod}(\mathrm{f})$.

Given arrows $f: \mathrm{Ob}_{1} \rightarrow \mathrm{Ob}_{2}$ and $g: \mathrm{Ob}_{2} \rightarrow \mathrm{Ob}_{3}$, i.e. with:

$$
\operatorname{cod}(f)=\operatorname{dom}(g)
$$


there is given an arrow:

called the composite of $f$ and $g$.

$$
g \circ f: O b_{1} \rightarrow O b_{3}
$$

To each object $O b_{1}$ there is given an arrow:

$$
1_{\mathrm{Ob} 1}: \mathrm{Ob}_{1} \rightarrow \mathrm{Ob}_{1}
$$

called the identity arrow of $O b_{1}$.

Then, for all pair of arrows in the which the object origin is target of another is possible combine an in agreement more long arrow shown in the diagram of the Figure 1.

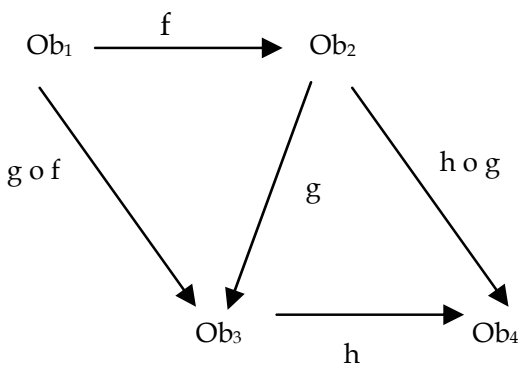

Fig. 1. Morphism of the category C

These data are required to satisfy the following laws:

- Associativity:

- Unit:

$$
h \circ(g \circ f)=(h \circ g) \circ f \text {, for all } f: O b_{1} \rightarrow O b_{2}, g: O b_{2} \rightarrow O b_{3}, h: O b_{3} \rightarrow O b_{4}
$$

$$
f \circ 1_{O b 1}=f=1_{O b 2} \circ f, \text { for all } \mathrm{f}: O b_{1} \rightarrow O b_{2}
$$

Definition 2: Category is equal $(\mathrm{Ob}, \mathrm{Mor})$ where $\mathrm{Ob}$ is the object of category and Mor is the morphism, satisfying:

- The morphism associates pairs of objects. A morphism should exist as $\operatorname{Mor}\left(\mathrm{Ob}_{1}, \mathrm{Ob}_{2}\right)$;

- The morphism composition is morphism;

- The morphism composition is associative;

- $\quad$ The identity morphism exists.

Definition 3: If the composition of the morphism $f$ with the morphism $g$ is equal the composition of the morphism $f$ with the morphism $h$ :

Then $f$ is a monomorphism:

$$
f \circ g=f \circ h \rightarrow g=h
$$

$$
\mathrm{Ob}_{1} \stackrel{\mathrm{g}}{\stackrel{h}{\longrightarrow}} \mathrm{Ob}_{2} \stackrel{f}{\longrightarrow} \mathrm{Ob}_{3}
$$

Definition 4: If the diagram is commutative, the composition of the morphism $g$ with the morphism $f$ is equal the composition of the morphism $h$ with the morphism $f$, that implies the morphism $g$ is equal the morphism $f$ : 
If

$$
g \circ f=h \circ f
$$

$$
g \circ f=h \circ f \rightarrow g=f
$$

Then, $f$ is an epimorphism:

$$
\mathrm{Ob}_{1} \stackrel{f}{\longrightarrow} \mathrm{Ob}_{2} \stackrel{\mathrm{g}}{\stackrel{h}{\longrightarrow}} \mathrm{Ob} b_{3}
$$

Definition 5: If the morphism $g$ is equal the morphism $h$ exists a monomorphism $(g=h)$. In the same way, $f$ is epimorphic if $f=k$. Therefore $f$ is an isomorphism because it is monomorphic and epimorphic, as shown the equation 13:

$$
\mathrm{Ob}_{1} \stackrel{g}{\stackrel{\mathrm{h}}{\longrightarrow}} \mathrm{Ob}_{2} \stackrel{f}{\longrightarrow} \mathrm{Ob}_{3} \stackrel{l}{\stackrel{\mathrm{k}}{\longrightarrow}} \mathrm{Ob}_{4}
$$

The correspondence of domain objects to another is produced by the morphism which preserves the defined characteristics in both domains (Barrett \& Mackaay, 2006). An important concept in this work is the context change, in others words, the category change, this can be done by a functor (Lambek \& Scott, 1986) that associates the category to the other categories.

Definition 6: A Functor it is the mathematical object that, given two categories, associates objects to objects and morphisms to morphisms, and that satisfy to the following conditions:

- The functors refer to pairs of categories. The properties of a specific AEHS can be associated other AEHS, for identification of objects and common properties of the both;

- An associative composition of functors that generates new functors exists. AEHS can be associated to compose connections that facilitate the reutilization of components;

- The identity functor that associates a category to it same exists. It allows defining exclusive characteristics of an AEHS for application in a specific domain that doesn't possess direct associations with other AEHS.

The functors, as well as the morphism, can be monomorphic, epimorphic and isomorphic.

\section{The proposed formalism for B-AEHS}

In general, the modeling of AEHS involves the student modeling, of the domain and adaptation. The letter (a) of Figure 2 shows an Educational Adaptive System composed by User Model, Domain Model and Interaction Model, similar to the classic system proposed by Benyon \& Murray (1993).

The User Model represents the Student Model that contains the generic and psychological profile of the user. The student's model is used as the basis of adaptation of the feature content and it should assist their objectives. In the adaptation model, after the specification of the models of the domain and of the student, these are combined for the process of generation of appropriate feature content through an adaptive interface. In the student modeling, besides the student's preference the knowledge state of the same ones should be defined.

The students' preferences are not limited only for the feature aspects, but also related to the content. Usually, the system maintains user's individual model as a layer of the model of the 
Cat $($ B-AEHS_Ob)

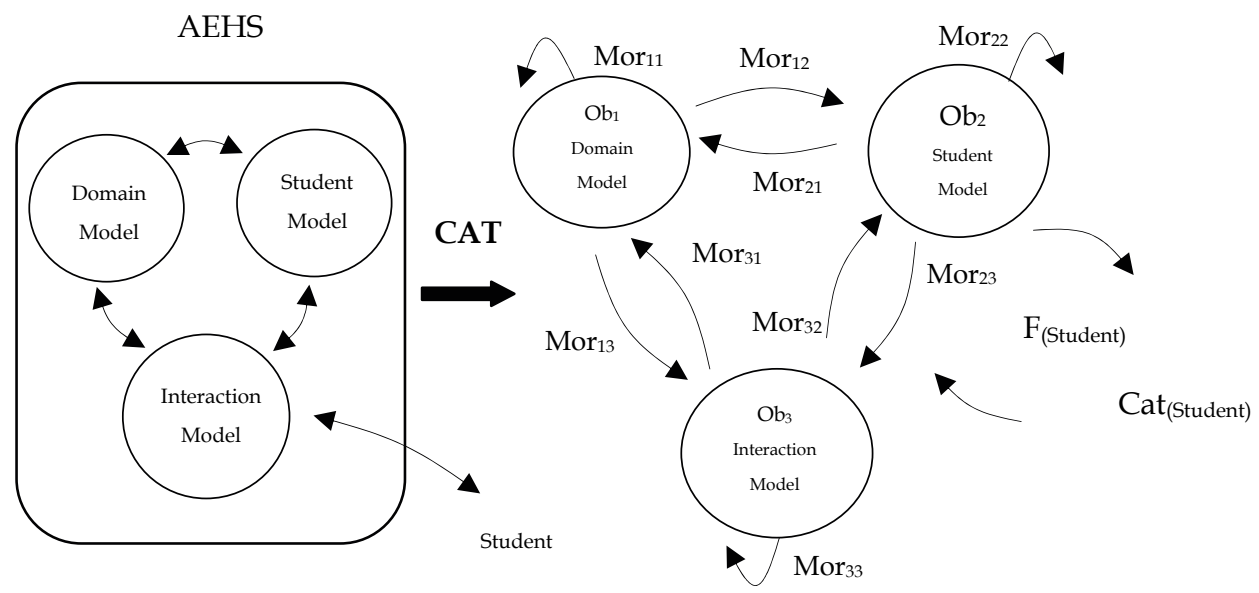

(a)

(b)

Fig. 2. The AEHS Model

domain to register the users related with the concepts of the domain current state. The Domain Model defines the main aspects of the system in the considered context to carry out the inferences. These aspects can be described in different levels such as Task Level, Physical Level and Logical Level (Benyon, 1993). Therefore, the domain model is the basis for all of the inferences and adaptations. The domain modeling that involves a specification of concepts and structure from crucial aspects of the system. The domain model is used to define which information will be processed in the application. The Interaction Model assures the dialogue between the user and application. It can register the precedent interactions in a Knowledge Base (Benyon, 1993). This model contains the mechanism to adaptation of the interface, inference of the user's properties and evaluation of the presented contents.

This AEHS model can be categorized considering each objects and their associations as morphisms of a category. A morphism allows specifying the courses and users' paths in AEHS. In a first abstraction, the modules AEHS are treated as objects that may or may not have associations with each other. The use of CT can facilitate the formal definition of these associations. We called the categorized model B-AEHS.

Given the three modules (student, domain and interaction), shown in section (a) of Figure 2. These modules can be categorized as objects $O b_{1}, \mathrm{Ob}_{2}$ and $O b_{3}$, as shown in section (b) of Figure 2. The categorization of the model can be made, therefore they are satisfied the following conditions:

- The morphism refer to pairs of objects: the morphisms Mor $_{12}, M_{21}$, Mor $_{23}, M_{0} r_{32}, M_{0} r_{13}$, $\mathrm{Mor}_{31}$ may associate the objects $\mathrm{Ob}_{1}$ (domain model), $\mathrm{Ob}_{2}$ (student model) and $\mathrm{Ob}_{3}$ (model of interaction) of AEHS;

- A composition of morphisms is morphism. The object $O b_{1}$ can be associated to the object $\mathrm{Ob}_{3}$ directly through the morphism Mor $_{13}$ or $M_{10} r_{31}$. These morphisms types allows identifying all of the paths traveled in AHS, in time of project, guaranteeing that 
there is not break of the flow of information and the user does not loss in the space of information in run time of the system.

- The composition of morphisms is associative: the morphisms allow visual identification of nodes and links regardless of the authoring tool or implementation. An example of composition of morphisms involving AEHS objects is given below:

$$
\begin{aligned}
& \text {Mor}_{12} \circ \text { Mor }_{23}=\text { Mor }_{31} \\
& \text {Mor}_{23} \circ \text { Mor }_{31}=\text { Mor }_{12} \\
& \text { Mor }_{31} \circ \text { Mor }_{12}=\text { Mor }_{23} \\
& \text { Mor }_{32} \circ \text { Mor }_{12}=\text { Mor }_{13} \\
& \text { Mor }_{13} \circ \text { Mor }_{32}=\text { Mor }_{21} \\
& \text { Mor }_{21} \circ \text { Mor }_{13}=\text { Mor }_{32}
\end{aligned}
$$

- $\quad$ The identity morphism must exist. The identity morphisms $M_{10} r_{11}, M_{12} r_{22}$ and $M^{\prime} r_{33}$ allow associations of the objects themselves. The user can decide, for instance, not to change of page in B-AEHS, or the own system, given an access of the user can not change the method of adaptive presentation.

- The association can also be made by the composition of the morphisms Mor $_{12}$ and Mor $_{23}$ or $M_{0 r}$ and $M_{20}$. The morphism allow the visual identification of links and nodes independently of the authorship tool or of the implementation.

Satisfied the categorical conditions, can be made formal representation:

- The properties of a specific B-AEHS can be associated to other for the identification of objects and common properties in both;

- B-AEHS can be associated to compose connections that facilitate the utilization of components;

- It is possible to define exclusive characteristics of a B-AEHS for application in a specific domain that does not have direct associations with other B-AEHS.

With this representation by morphisms and objects can be defined associations between the components of B-AEHS. For a model that involves a change of context or external for the object modeling system uses the concept of functors. In terms of domains transformations of domains, B-AEHS can be modeled categorically as:

$$
\mathrm{Cat}_{(B-A E H S)}=\left(O b, F_{t}\right)
$$

Where $O b$ are objects of the category $B$-AEHS and $F_{t}$ are functors that associate the objects of the category $\mathrm{Cat}_{(\mathrm{B}-\mathrm{AEHS})}$ with it same or with other categories, as for instance, a category of users $\mathrm{Cat}_{\text {(Student). }}$. This approach can be interesting to find universal properties of the systems, in different domains and applications.

In the case of specification of a B-AEHS, CT can be applied to define the user's models, of the domain and of the adaptation defining the associations among each module of the system. It is possible to use a functor forget (Almeida, 2002) that defines the unique characteristics of a system for application in a specific area that has no direct associations with other systems. Thus, on B-AEHS specification, the CT can be used at all levels. For example, it is possible to identify categories of B-AEHS, domain models, user models and 
models of adaptation. It is possible also categorize only the objects and sub objects of different B-AEHS. This approach allows describe the relationships between systems and systems users and systems.

According the conceptual modeling, new objects can be defined and the conditions categories can be used to reduce the ambiguities of the system. The concepts presented here are extensible for any AEHS, because the categorical representation is independent of platform, number of objects and associations between them. The formal treatment can be given in any level of abstraction of the system.

For the design of an adaptive interface the Neuroanatomy system (B-AEHS) was divided into three modules (the user model, domain model and interaction model). The model of interaction was categorized so that each page was treated formally as an object and its components as sub-objects. Project-level navigation was chosen formalism more appropriate to simplify the specification as shown in the following sections of work.

\subsection{The direct guidance}

Direct guidance (Brusilovsky, 2004) is the simplest technology of adaptive navigation support. Direct guidance suggests the "next best" node for the user to visit according user's goals, knowledge, or/and other parameters represented in the user model. So that to provide direct guidance, an adaptive educational hypermedia system (AEHS) usually presents an additional dynamic link (Brusilovsky, 2004). From a given node, the system generates a link for more appropriate node, which is also given a link to another node most appropriate and so on. It is applied to decide which one is the next step the user must follow.

So that to categorize the Direct guidance is the use of the categorical concepts of the categorical Determination Problem (Lawvere \& Schanuel,1997). The Figure 3 presents the categorical mapping for Direct guidance made by determination. If morphism $f$ is given, each $g$ can be obtained by $h=g \circ f$ composition. Therefore, given a set of known links $O b_{1}$ for Direct guidance is possible to compose these links for association with another set of nodes $\mathrm{Ob}_{2}$, to compose the path of the navigation. Assuming the existence of a morphism $f$ that maps $\mathrm{Ob}_{1}$ in $\mathrm{Ob}_{2}\left(\mathrm{Ob}_{1} \stackrel{f}{\rightarrow} \mathrm{Ob}_{2}\right)$ and a set of links $\mathrm{Ob}_{3}$ in the adaptive navigation. Then each morphism $g$ of $\mathrm{Ob}_{2}$ to the $\mathrm{Ob}_{3}$ can be composed with $f$ for generate the path for the user model by mapping $\mathrm{Ob}_{1} \rightarrow O b_{3}$. Therefore, $f$ maps $\mathrm{Ob}_{2}$ in $\mathrm{Ob}_{3},\left(\mathrm{Ob}_{2} \rightarrow O b_{3}\right)$ and also offers the mapping $\mathrm{Ob}_{1} \rightarrow \mathrm{Ob}_{3}$.

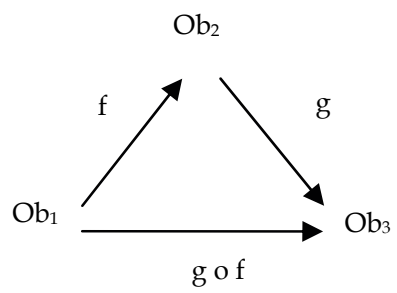

Fig. 3. Model of Direct Guidance by Determination Problem

Another way to categorize the Direct guidance is to use the constant morphism as showed in section 5 . 


\subsection{Adaptive link sorting}

Rather than provide the best link to the direct guidance, this technique offers a list of links in descending order of relevance for the user. Refers to the order in which the adaptive links are presented to the user according its relevance. The ordination may be a similarity, prerequisite, relevance, knowledge of the user, etc. The ordering of content is made in accordance with the user profile. From the node most important links are classified according to the user model, after being presented in descending order. In what order the links should be submitted? CT can be used to model the sort of links. Figure 4 presents a set of links that should be classified according the relevance R.

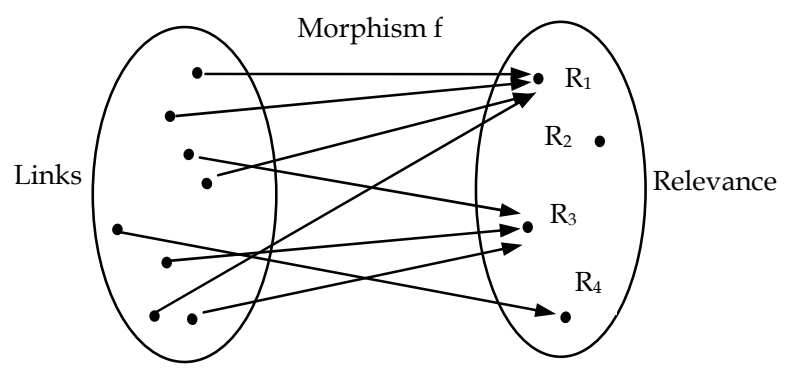

Fig. 4. Model of sort by relevance (adapted from Lawvere \& Schanuel (1997)).

The classification can be made by a property (Lawvere \& Schanuel,1997). As shown in Figure 5, assuming that $\mathrm{Ob}_{2}$ has three elements that they represent different relevance assignments. Then, without change the morphism $f$ is possible rearrange the elements of $O b_{1}$ in three different classifications according to the user's model: ordering links for the user basic level, ordering of links for intermediary user level and ordering links for user advanced level. The classification consists of placing in the same group all the elements of $O b_{1}$ that go to the same element of the $O b_{2}$. The links are divided into fibers according to relevance $R_{1}, R_{2}$ and $R_{3}$. Therefore, a mapping $O b_{1} \rightarrow O b_{2}$ produces a structure in $O b_{1}$ domain and when we want to emphasize that the mapping effect is referred as the valuation property of the set of links $\mathrm{Ob}_{2}$.

For a general mapping is possible to say that the morphism $f$ ranks (or orders) $O b_{1}$ in $O b_{2}$ or that the morphism $f$ is a classification of $O b_{1}$ by $O b_{2}$. This condition is valid if $\mathrm{Ob}_{2}$ consists of numbers. Since $f$ is given, each element $o b_{2}$ of $O b_{2}$ determines which elements of the set of links $O b_{1}$ are classified by $o b_{2}$.

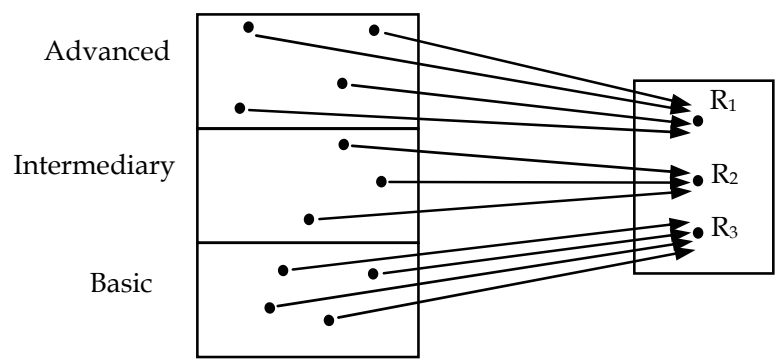

Fig. 5. Sort links by property (adapted from Lawvere \& Schanuel (1997)) 
The categorization of the classification of links can be made by pullback of two morphisms, as shown in Figure 6.

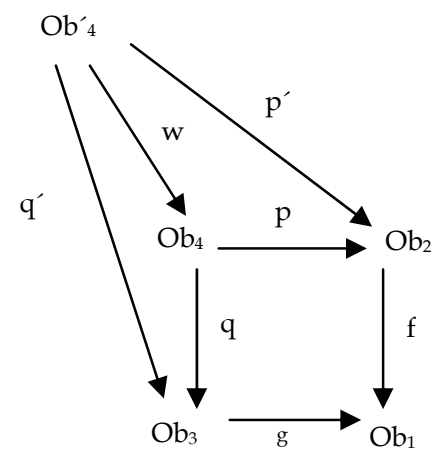

Fig. 6. Link classification by Pullback

Definition 7. The pullback is a limit of a diagram, constructed by two morphisms with the same target object (Lawvere \& Schanuel,1997). Given two morphisms $f: O b_{2} \rightarrow O b_{1}$ and $g$ : $\mathrm{Ob}_{3} \rightarrow O b_{1}$, the pullback $\mathrm{Ob}_{4}$ is given by the pair of morphisms $p: \mathrm{Ob}_{4} \rightarrow \mathrm{Ob}_{2}$ and $q: \mathrm{Ob}_{4} \rightarrow O b_{3}$ such that the diagram commutes:

$$
f \circ p=g \circ q
$$

Since for all objects $O b_{4}^{\prime}$ and all morphisms $p^{\prime}: O b_{4}^{\prime} \rightarrow O b_{2}$ and $q^{\prime}: O b_{4}^{\prime} \rightarrow O b_{3}$ such that:

$p^{\prime} \circ f=q \circ g$ exists a unique morphism $w: O b_{4}^{\prime} \rightarrow O b_{4}$ such that $q \circ w=q^{\prime}$ and $p \circ w=p^{\prime}$. Each relevant $R$ must be considered as a target.

\subsection{Adaptive link generation}

In order to generate new links of interest to the user on the information network that they had not been defined in the authorship. The link generation includes three cases: discovering new useful links between documents and adding them permanently to set existing links; generating links for similarity-based navigation between items; and dynamic recommendation of relevant links (Brusilovsky, 2004). How interesting links can be generated? The generation of links can be categorized by categorical product which is a structural generalization of the concept of Cartesian product.

Definition 8. The Cartesian product $O b_{1} \times O b_{2}$ of the objects $O b_{1}$ and $O b_{2}$ consists of ordered pairs $<o b_{1}, o b_{2}>$ where $o b_{1} \in O b_{1}, o b_{2} \in O b_{2}$ and there are projections $\pi: O b_{1} \times O b_{2} \rightarrow O b_{1}$ and $\pi^{\prime}: \mathrm{Ob}_{1} \times \mathrm{Ob}_{2} \rightarrow \mathrm{Ob}_{2}$.

\subsection{Adaptive link hiding}

The purpose of navigation support is hide and restrict the navigation space by hiding, removing, or disabling links that go to irrelevant pages. A page can be considered irrelevant for several reasons: for example, if it is not related to the user's current learning goal or if it presents materials which the user is not yet prepared to understand. Hiding protects users from the complexity of the whole hyperspace and reduces their cognitive overload (Brusilovsky, 2004). The categorial adaptive of the link hiding can be represented as a 
Choice Problem (Lawvere \& Schanuel,1997). The links that are hidden are chosen given a rule disabling a set of links selected as shown in Figure 7.

Considering that $O b_{3}$ is a set of links are hidden in the adaptive presentation, $O b_{1}$ is the set of all links and $h$ the morphism of the $O b_{1}$ to $O b_{3}$ that determines the concealment of the links. Therefore taking $\mathrm{Ob}_{2}$ as the set of all rules of deactivation, the problem is to find the morphism $f$ that associates disabling link in accordance with its rule on the set $\mathrm{Ob}_{3}$ by the morphism $g$.

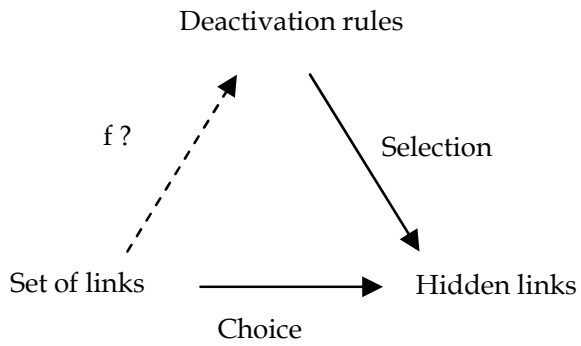

Fig. 7. Hiding links by deactivation rules

Figure 8 shows the categorization of hiding links. In order to find a morphism $f$ such that $g$ - $f=h$, must be chosen for each element $o b_{1}$ of $O b_{1}$ an element $o b_{2}$ such that $g\left(o b_{2}\right)=h\left(o b_{1}\right)$.

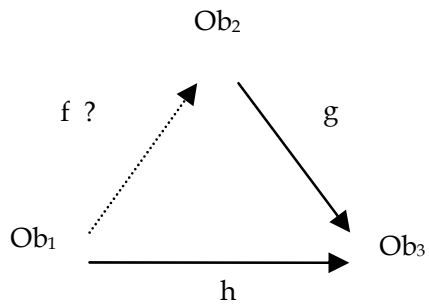

Fig. 8. Categorization of the hiding links

\subsection{Map adaptation}

This technique includes several forms of adaptation of maps to local and global hypermedia shown to the user, applied in a graphic display of the navigation structure (Brusilovsky, 2004). Maps (local and / or global) and indexes are presented for easy navigation. How to represent the maps and indexes? Map adaptation (Brusilovsky, 2002) can be modeled categorically defining sub-objects. The sub-object is the categorical version of subset in set theory (Lawvere \& Schanuel,1997). Is defined as the subset of objects $O b_{1} \subseteq O b_{2}$ as a monomorphism $\mathrm{f:O} \mathrm{b}_{3} \rightarrow \mathrm{Ob}_{2}$.

Definition 9. If the composition of the morphism $f$ with morphism $g$ is equal to the composition of the morphism $f$ with morphism $h$ :

$$
f \circ g=f \circ h \rightarrow g=h
$$

Then $f$ is a monomorphism. 
Figure 9 shows a diagram of a monomorphism for mapping the routes driven by links. The paths (represented by the composition of morphisms) are equivalent when they lead to the same link, independently of the user navigation point.

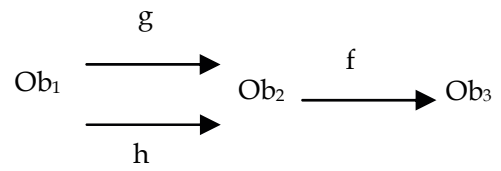

Fig. 9. Monomorphism

Figure 10 shows the equivalent diagram shown in Figure 9. The maps are produced as subobjects of the category of nodes for guide the user in defined pathways.

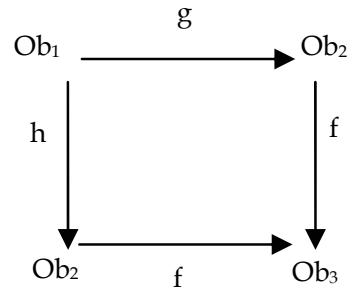

Fig. 10. Diagram equivalent mapping

Let $C$ a category. If $f: b \rightarrow a$ and $g: c \rightarrow a$ are two arrows with a common target, then it is said that $f \leq g$ if and only if exists $h: b \rightarrow c$ such that $g \circ h=f$. If $f \leq g$ and $g \leq f$ then we say that $f \leftarrow g$ is an equivalence relation between monomorphisms which have a common target (Lawvere \& Schanuel,1997). The indexes can be modeled as amalgamated sum (pushout) as shown in Figure 11.

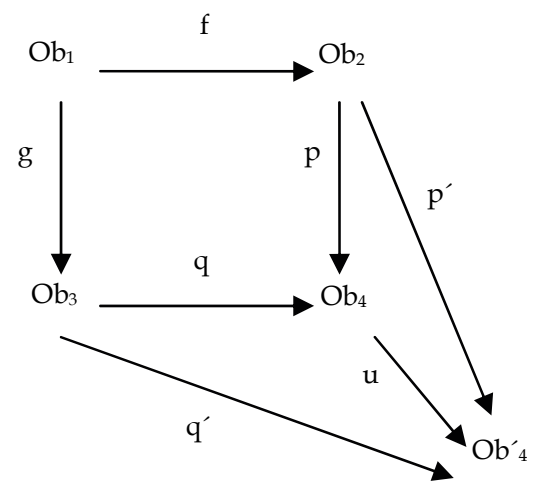

Fig. 11. Pushout of two morphisms $\mathrm{f}$ and $\mathrm{g}$

Definition 10. The amalgamated sum (pushout) is the colimit of a diagram consisting of two morphisms with the same source object (Lawvere \& Schanuel, 1997). Given the morphism $f$ : $O b_{1} \rightarrow O b_{2}$ and the morphism $g: O b_{1} \rightarrow O b_{3}$, the pushout $O b_{4}$ is obtained by the pair of morphisms $p: \mathrm{Ob}_{2} \rightarrow \mathrm{Ob}_{4}$ and $q: \mathrm{Ob}_{3} \rightarrow \mathrm{Ob}_{4}$ such that the diagram commutes. 


$$
p \circ f=q \circ g
$$

Since for all objects $O b_{4}^{\prime}$ and all morphism $p^{\prime}: O b_{2} \rightarrow O b_{4}^{\prime}$ and $q^{\prime}: O b_{3} \rightarrow O b_{4}^{\prime}$ such that $p^{\prime} \circ f=q^{\prime} \circ g$ exists unique morphism $u: O b_{4} \rightarrow O b_{4}^{\prime}$ such that $\mathrm{u} \circ \mathrm{q}=\mathrm{q}^{\prime}$ and $u \circ p=p^{\prime}$.

The amalgamated sum is dual concept of the fibered product (pullback) (Fiadeiro, 2005). Thus, an index is a point to which converge the various links of the system. In an adaptive interface, the categorization enhances the effect of construction of indexes according to the user model.

\subsection{Adaptive link annotation}

The links are commented to show its relevance, i.e., the anchors have a different aspect visible to show the relevance of the destination. Different modifications are performed in a link in order to increase their information, informing to the user what will come in the next nodes.

The aggregation of links to more information is given to providing more information about the target nodes of the links.

The annotations can be textual, visual (icons, colours or font size) (Brusilovsky, 2007). How to represent more information to the links? The adaptive annotation of links can be represented categorically with the sum as shown in Figure 12.

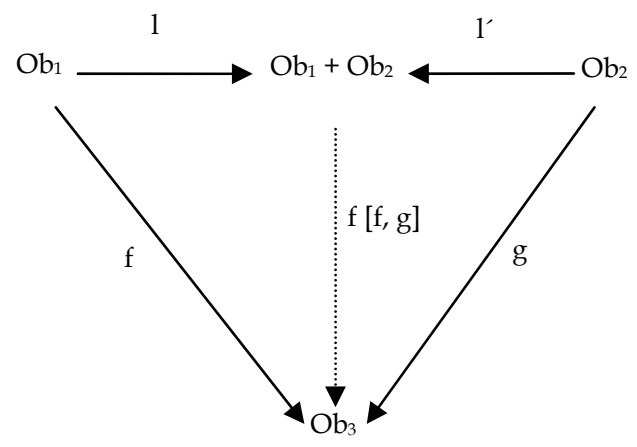

Fig. 12. Sum of objects

Definition 11. The sum or co-product is the dual concept of product. In sum concept the morphisms are called inclusions (Jay, 1993). Considering the objects $O b_{1}$ and $O b_{2}$ in a Category $\mathrm{C}$. They have sum if the object formed by $\mathrm{Ob} b_{1}+\mathrm{Ob}_{2}$ is endowed with injections.

$$
\mathrm{Ob}_{1} \stackrel{\mathrm{lOb} b_{1} \cdot \mathrm{Ob}_{2}}{\rightarrow} \mathrm{Ob} b_{1}+\mathrm{Ob}_{2} \stackrel{\mathrm{l}^{\prime} \mathrm{Ob}}{\stackrel{\mathrm{Ob}}{2}} \stackrel{\mathrm{Ob}}{\leftarrow}
$$

For each object $O b_{3}$ and the pair of morphisms $f: O b_{1} \rightarrow O b_{3}$ and $g: O b_{2} \rightarrow O b_{3}$ exists a unique morphism $[f, g]: O b_{1}+O b_{2} \rightarrow O b_{3}$ making the diagram commutative.

Figure 13 shows that considering on the link annotation modeling the pair of morphisms $\mathrm{Ob}_{1} \stackrel{a}{\rightarrow} \mathrm{Ob} b_{3}, \mathrm{Ob}_{2} \stackrel{b}{\rightarrow} \mathrm{Ob} b_{3}$ in a category is the sum of the $\mathrm{Ob} b_{3}$ and $\mathrm{Ob} b_{2}$, if each object of the $\mathrm{Ob}_{4}$ and each pair $\mathrm{Ob} b_{1} \stackrel{c}{\rightarrow} \mathrm{Ob}_{4}, \mathrm{Ob}_{2} \stackrel{d}{\rightarrow} \mathrm{Ob}_{4}$ is exactly an map $\mathrm{Ob} b_{3} \stackrel{e}{\rightarrow} \mathrm{Ob}_{4}$ to both 
morphisms $c=e \circ a$ and $d=e \circ b$. The morphisms $a$ and $b$ are called morphisms injection of the sum representing the modeling will be presented to user through aggregations made in sets of links, represented by $\mathrm{Ob}_{1}$ and $\mathrm{Ob}_{2}$.

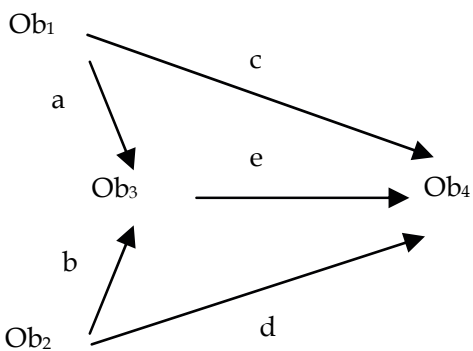

Fig. 13. Categorization of the adaptive link annotation

\section{Formal modeling of a Neuroanatomy tutorial}

The following sub-sections present the main techniques used in a project of a Neuroanatomy tutorial. Figure 14 shows the screen of an interactive system, called Virtual Laboratory of Neuroanatomy (VLN), developed at Pontifical Catholic University of Minas Gerais, Brazil.

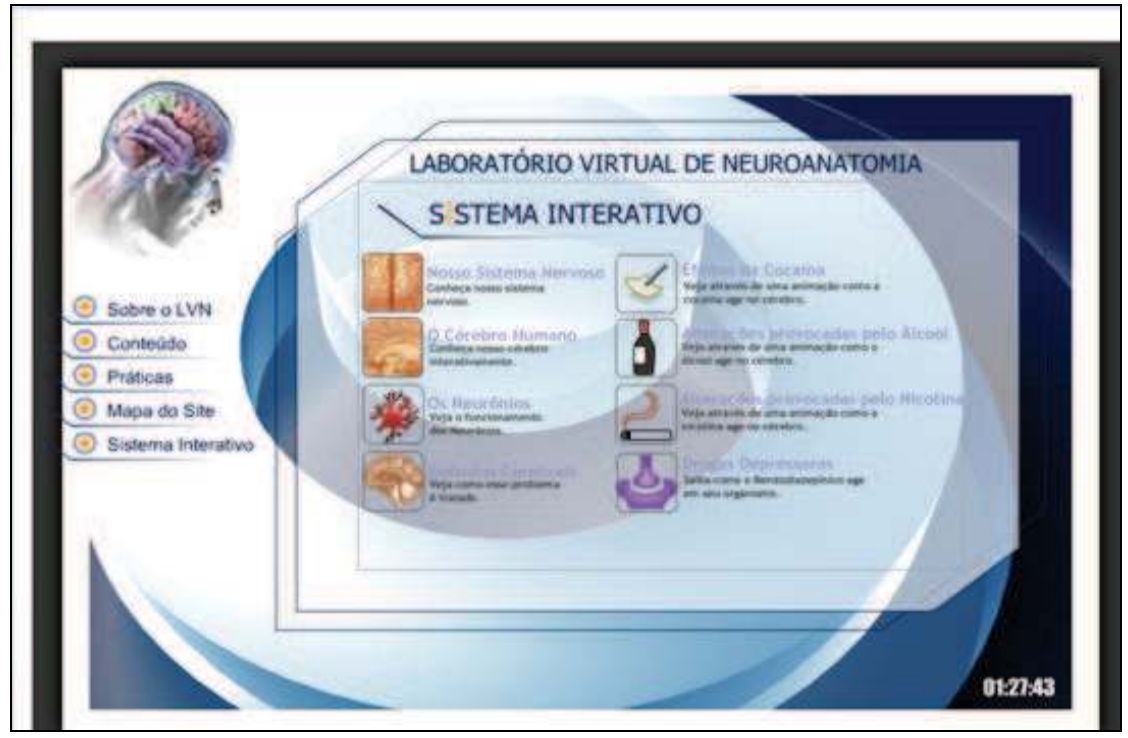

Fig. 14. Screen of the Neuroanatomy Educational System

The system formed the basis for the design of adaptive navigation treated in this work. The main pages of the system were treated as objects. The main parts of system structure were categorized into four distinct objects:

- $\mathrm{Ob}_{1}$ - page presentation of the VLN, 
- $\mathrm{Ob}_{2}$ - Content

- $\mathrm{Ob}_{3}$ - Practice

- $\mathrm{Ob}_{4}$ - Site Map e;

- $\mathrm{Ob}_{5}$ - Interactive System.

The goal of the study was to development the system parts using a formal method for abstraction in high level. The part projected consists of an agent (robot) that presents the drugs effect in nervous system and then presents a quiz to the student.

The chosen methodology was to project of the adaptive navigation support. The adaptive navigation helps users to follow the paths in hyperspace by adapting the form of presentation of the links in the hypermedia network.

The categorization is used in this work to model the actions related to adaptation of navigation, which is to change the navigation structure or in how this structure is presented to the user.

The adaptive navigation helps users to follow the paths in hyperspace by adapting the form of presentation of the links in the hypermedia network.

Figure 15 shows representations of direct guidance, sorting and generation of links in educational contexts in the VLN screen. For simplicity, we consider only the design of a single page (page 5) of an interactive system, specified as the object $\mathrm{Ob}_{5}$. This page presents a quiz to the student.

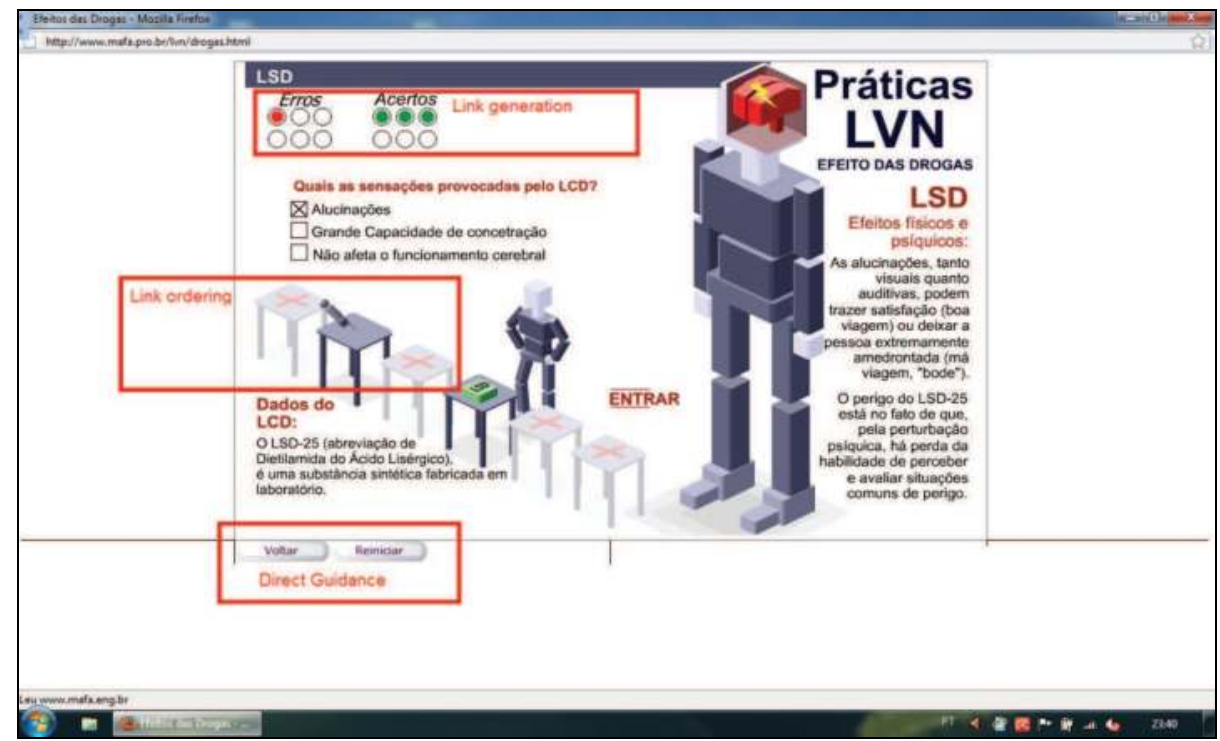

Fig. 15. Links of Adaptive navigation in VLN

Considering each page as an object of the category page and each object (links, images, actions) as sub objects of the category page is possible to refine the structure. The letter (a) of Figure 16 shows the direct guidance in modeling categorical one page of practice of a Virtual Laboratory of Neuroanatomy (VLN). In a first abstraction, formalization of direct guidance offered by the CT through the mapping done by a constant morphism. Considering $\mathrm{Ob}_{3}$ as an only target node is equivalent to an only choice for user direct guidance in the AEHS, if 
exist a morphism $g$ that maps $\mathrm{Ob}_{2}$ to $\mathrm{Ob}_{3}$, the composition $h=g \circ f$ will send all of the elements of $O b_{1}$ to $O b_{3}$ to form the structure of the presentation of the links in the navigation.

Let is suppose that $\mathrm{Ob}_{2}$ is a one-element set, so $f$ is already known: it takes all elements of $O b_{1}$ to the only element of $O b_{2}$. A map $h$ must send all elements of $O b_{1}$ to the same element of $\mathrm{Ob}_{3}$.

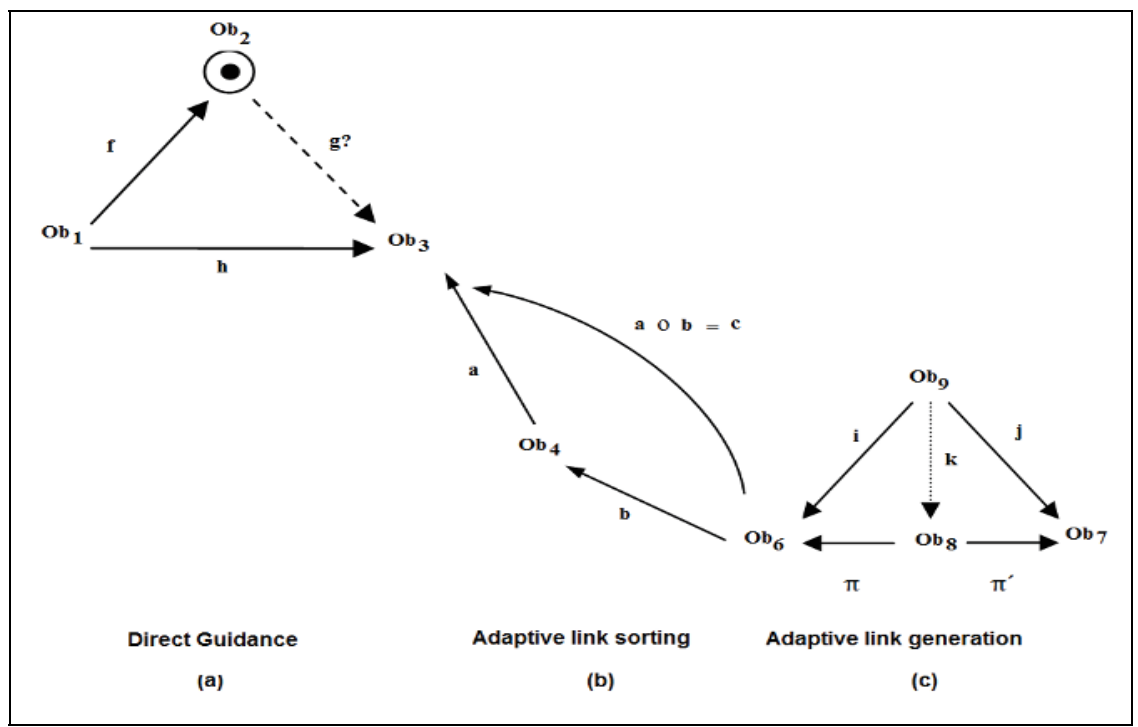

Fig. 16. Categorical denotation of navigation support in a VLN screen page

The letter (b) of Figure 16 shows the specification of the ordering of links in the page object of Practice Interactive System. The morphism classifies all links; each link determines the relevance and the relevant links are then sorted again to form the paths of adaptive navigation. If $\mathrm{Ob}_{6}$ is the set of all links and $\mathrm{Ob}_{4}$ is the set of all relevant values assigned in accordance with the user level. Then the morphism $\left(\mathrm{Ob}_{6} \rightarrow \mathrm{bb}_{4}\right)$ assigns each link with a relevance.

The letter (c) of Figure 16 shows the generation of new links of interest to the users that were not defined at the time of authorship in information network. The modeling to link generation was formalized by categorical product.

As shown in Figure 16 the object $O b_{8}$ is considered as a new link (or set of links) generated. Considering that $\mathrm{Ob}_{6}$ and $\mathrm{Ob}$ are objects of the category $\mathrm{C}$ ("Question and Answers"), the product of $O b_{1}$ and $O b_{2}$ is given by an object $O b_{4}$ and the pairs of morphisms $\pi: O b_{4} \rightarrow O b_{1}$ and $\pi^{\prime}: \mathrm{Ob}_{4} \rightarrow \mathrm{Ob}_{2}$ called first and second projection, respectively. For each object $\mathrm{Ob}_{9}$ and the pair of morphisms $i: \mathrm{Ob}_{9} \rightarrow \mathrm{Ob}_{6}$ and $\mathrm{j}: \mathrm{Ob}_{9} \rightarrow \mathrm{Ob}_{7}$ there is a unique morphism $k: \mathrm{Ob}_{9} \rightarrow$ $\mathrm{Ob}_{8}$ such that the diagram is commutative. The object $\mathrm{Ob}_{4}$ is considered as a new link (or set of links) generated.

The diagram commutes if each pair of paths through the diagram is such that they have the selfsame start and end points defining a same morphism. Therefore, the diagram in letter (c) of Figure 16 we have: 


$$
\begin{gathered}
\pi \circ k=i \\
\pi^{\prime} \circ k=j
\end{gathered}
$$

The CT showed to be a useful method for modeling the characteristics of the VLN adaptive navigation. The use of CT allowed more complex representations in topological space. To design the structure of links and of nodes, CT seemed a more natural approach.

Figure 17 presents the equivalent model of the Figure 16.

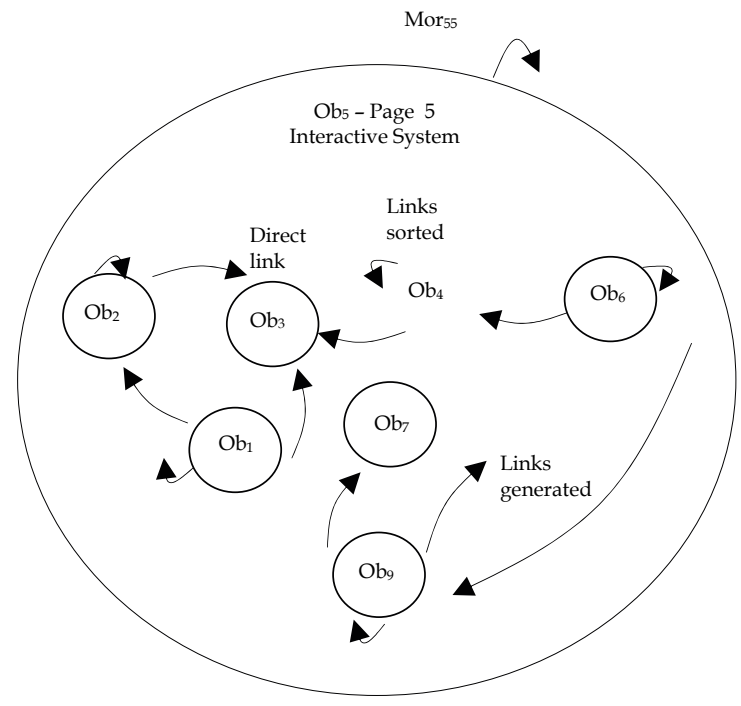

Fig. 17. A simple diagram equivalent for denotation of navigation support for page 5

A categorical model of AEHS aims to represent high-level connections that can be made between the components of an educational hypermedia system. For instance, the identity morphisms may represent, for example, the links that associate the page to themselves, the behavior of the user can decide not to change the page in the AEHS, the system behavior not changes the method of presentation adaptive to the student, etc.

The diagram of Figure 17 was used to describe the pathways of the B-AEHS for Neuroanatomy tutorial. It was possible to reduce the problems with navigation. The results showed that the great advantage of using CT was to provide a high degree of generalization to the conceptual representation of the system. The level of abstraction and generality offered by CT allows its use in the development of many different models AEHS. The decomposition of the system leads to breaking up large specifications into components that can be refined independently with the composition of combinations that must meet a higher specification.

The system was viewed as a whole however their parts were built separately. The formal model of adaptive navigation support simplified the structure of links reducing the problems of orientation while maintaining the degree of freedom in navigation. 


\section{Conclusion}

In all architectures exists the general consensus that a model of AEHS should contain minimal a student model, a domain model, an adaptation model and interaction mechanisms with the user. AEHS can adapt its behavior for the user or the context of the considered domain. A construction of students' models usually requests that are made a lot of suppositions on the same ones: abilities, knowledge, needs, or preferences, as well as its behavior and interaction with the system. Besides, a consensus of the specialists exists, mainly, to that are devoted applied AEHS to the education area that the system should consider the user's cognitive aspects.

Many studies are dedicated to the study of quality of Health Education Systems. However, much of the literature studies are focused on teaching (Kosone, 2009), learning strategies (Patel et al, 2009), usability ( $\mathrm{Ng}$ et al., 2002), etc. No importance is given to studies on the construction of educational programs in this area.

This work emphasizes that the use of categorical techniques can contribute to the quality of these systems because a great benefit of the use of formal methods is to reduce the number of errors in systems.

Several conventional systems and adaptive hypermedia have been developed without the use of modelling techniques, did not follow a formal methodology for implementations. Due to the numerous applications of these systems and the development of hypermedia technology models have emerged to represent references and arbitrary combination of mechanisms for specification of systems. There are few studies of adaptive navigation support in educational hypermedia systems in Biomedical Education. In modelling the characteristics of adaptive navigation the CT was applied to provide formalisms useful in defining the interconnections between the links. The adaptive navigation treats of the definition of the spatial layout and information related to the user interface (Brusilovsky, 2007). The use of CT allows more complex representations in topological space used to model adaptive navigation in AEHS context.

An important contribution of CT is to illustrate the formal mapping among different levels of the architecture of the program. In others words, there is the concept of the components generalization of low level in the programs architecture. Although any theory can be used for to define the objects of multiple levels of the B-AEHS architecture, compared with other theories, reduces the project complexity when different levels for schemata and diagrams are necessary.

Another advantage is that, as CT is based in diagrams, this primitive concept is most natural for definition of the dynamic and static aspects of B-AEHS model. This approach can be interesting to find universal properties of the systems, in different levels and modules.

The model design methods provide a systematic and consistent set of steps that assist the development of hypermedia systems. The use of CT can complement these methods simplify the modeling process. CT can offer a high level of abstraction for languages of description of AEHS architectures.

Finally, from our analysis, we concluded that CT has a rich symbolism that allows quickly visualize complicated facts and connections to model Adaptive Educational Hypermedia System by diagrams.

Future works can build CASE (Computer-Aided Software Engineering) tools to modeling BAEHS in the several biomedical areas. The CT treats of objects and its associations, therefore the tool can incorporate the benefits of the object-orientation and the usage of visual diagrams easily. 


\section{References}

Almeida, M. A. F. ; Azevedo, F. M. (2008). B-AEHS: a formal model for adaptive educational hypermedia system in a Biomedical Project evaluation, 4th European Conference of the International Federation for Medical and Biological Engineering, pp. 2734-2737, ISBN 978-3-540-89207-6, November, Springer Berlin Heidelberg, Antwerp, Belgium.

Almeida, M. A. F. (2002). Hipertômatos na Computação aplicada à Educação, PhD thesis, Federal University of Santa Catarina, Florianopolis.

Adamek, J.; Herrlich, H. \& Strecker, G.E. (2004). Abstract and Concrete Category: the Joy of Cats, John Wiley \& Sons, ISBN 9780471609223, New York, USA.

Arbib, M.; Manes, E. (1975) Arrows, Structures, and Functions - The Categorical Imperative. Academic Press, Inc., ISBN 0-12-059060-3, New York, USA.

Awodey, S. (2006). Category Theory, Oxford University Press Inc, ISBN 0-19-856861-4, New York, USA.

Barrett, J. W. \& Mackaay, M. (2006). Categorical representations of categorical groups, Theory and Applications of Categories, Vol. 16, No. 20, pp. 529-577, ISSN 1201-561X.

Benyon, D. ; Murray, D. (1993). Applying User Modelling to Human-Computer Interaction Design. Artificial Intelligence Review, Vol. 7, No. 3-4, pp. 199-225, ISSN 0269-2821.

Brusilovsky, P. (1996). Methods and Techniques of Adaptive Hypermedia, Journal of User Modelling and User-Adapted Interaction, Vol. 12, No. 6, pp. 87-129.

Brusilovsky, P. (2001). Adaptive hypermedia, User Modelling and User Adapted Interaction, Vol. 11, No. 1, pp. 87-110, ISSN 0924-1868.

Brusilovsky, P. (2002). Domain, task, and user models for an adaptive hypermedia performance support system, Proceedings of the 7th international Conference on intelligent User interfaces (IUIO2), pp. 23-30, ISBN 1-58113-459-2, January, ACM Press, San Francisco, USA.

Brusilovsky, P. (2004). Adaptive navigation support: from adaptive hypermedia to the adaptive web and beyond, PsychNology Journal, Vol.2, No. 1, pp. 7-23, ISSN 17207525.

Brusilovsky, P. \& Millán, E. (2007). User models for adaptive hypermedia and adaptive educational systems, The Adaptive Web Methods and Strategies of Web Personalization, Vol. 4321, pp.3-53, Springer Berlin Heidelberg, ISSN 0302-9743.

Campbell, B. ; Goodman, J.M. (1987) HAM: A General-Purpose Hypertext Abstract Machine In: Hypertext' 87 Proceedings, ACM, 1987, pp. 21-32.

De Bra, P.; Houben, G \& Kornatzky, Y (1993). Browsing Semantics in the Tower model. In: Computing Science Notes, Prentice-Hall.

Fiadeiro, J. L. (2005). Categories for Software Engineering, Springer Berlin Heidelberg, ISBN 3540-20909-3, New York.

Furuta, R. \& Scotts, P. (1990). The Trellis Hypertext Reference Model, In: Proceedings NIST Hypertext Standardization Workshop.

Ginderen, B. V. (1990). An Object-oriented Hypertext System for Computer-Aided Learning. Master's thesis, Eindhoven University Technology.

Gunawardena, J. (1996) New Connections between Mathematics and Computer Science, Technical Report 96002, Hewlett Packard Labs, Bristol, UK.

Halasz, F. ; Schwartz, M. (1994) The Dexter Hypertext Reference Model, Communications of the ACM, vol. 37 . 
Hardman, L. ; Bulterman, D. C.A \& Van Rossum, G. (1994) The Amsterdam Hypermedia Model: adding time and context to the Dexter model, Communications of the ACM, vol. 37, pp. 50-52.

Jay, C. B. (1993). An introduction to Categories in Computing, University of Technology, Sidney, Australia.

Kosonen, A. L. ; Haapala, I.; Kuurala, S. ; Savonlinna, S. M.; Hanninen, O. ; Carvalho, G.S. (2009). Health knowledge construction and pedagogical style in Finnish health education textbooks, Health Education, vol. 109, no. 3, pp. 226-241.

Lawvere, F. W. \& Schanuel, S. H. (1997). Conceptual Mathematics: a first introduction to categories, Cambridge University Press, ISBN 0-521-47249-0, New York, USA.

Lambek, J, Scott, P J (1986) Introduction to higher order categorical logic. Studies in Advanced Mathematics, Cambridge, Cambridge University Press.

Ng, M. H., Hall, W., Maier, P. and Armstrong, R. (2002) The Application and Evaluation of Adaptive Hypermedia Techniques in Web-based Medical Education. Association for Learning Technology Journal, 10 (3). pp. 19-40.

Pagano, R. L. (1992) Computer Simulation as an Educational Tool, PhD thesis, Faculty of Applied Sciences, University of Louvain la Neuve, Belgium.

Patel, V. L. ; Yoskowitz, N.A.; Arocha, J. F., Shortliffe, E. H. (2009) Cognitive and learning sciences in biomedical and health instructional design: a review with lessons for biomedical informatics education, Journal of Biomedical Informatics, ISSN: 1532-0464, vol. 42, pp. 176-197.

Rossi, G., Schwabe, D., Lyardet, F.(2010) Web Application Models Are More than Conceptual Models, Advances in Conceptual Modelling Lecture Notes in Computer Science, 2010, Volume 1727/2010, 239-252, DOI: 10.1007/3-540-48054-4_20. 


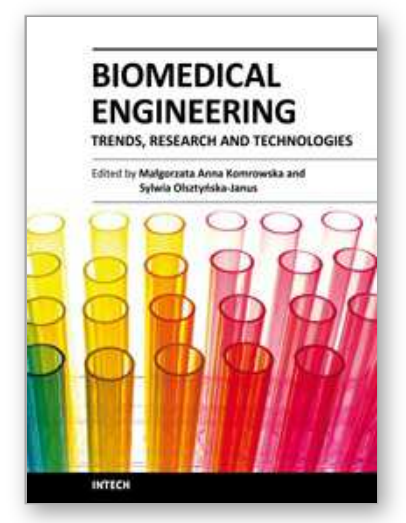

\author{
Biomedical Engineering, Trends, Research and Technologies \\ Edited by Dr. Sylwia Olsztynska
}

ISBN 978-953-307-514-3

Hard cover, 644 pages

Publisher InTech

Published online 08, January, 2011

Published in print edition January, 2011

This book is addressed to scientists and professionals working in the wide area of biomedical engineering, from biochemistry and pharmacy to medicine and clinical engineering. The panorama of problems presented in this volume may be of special interest for young scientists, looking for innovative technologies and new trends in biomedical engineering.

\title{
How to reference
}

In order to correctly reference this scholarly work, feel free to copy and paste the following:

Maria Aparecida Fernandes Almeida and Fernando Mendes de Azevedo (2011). Biomedical Adaptive Educational Hypermedia System: a Theoretical Model for Adaptive Navigation Support, Biomedical Engineering, Trends, Research and Technologies, Dr. Sylwia Olsztynska (Ed.), ISBN: 978-953-307-514-3, InTech, Available from: http://www.intechopen.com/books/biomedical-engineering-trends-research-andtechnologies/biomedical-adaptive-educational-hypermedia-system-a-theoretical-model-for-adaptivenavigation-suppor

\section{INTECH}

open science | open minds

\section{InTech Europe}

University Campus STeP Ri

Slavka Krautzeka 83/A

51000 Rijeka, Croatia

Phone: +385 (51) 770447

Fax: +385 (51) 686166

www.intechopen.com

\section{InTech China}

Unit 405, Office Block, Hotel Equatorial Shanghai

No.65, Yan An Road (West), Shanghai, 200040, China

中国上海市延安西路65号上海国际贵都大饭店办公楼 405 单元

Phone: +86-21-62489820

Fax: $+86-21-62489821$ 
(C) 2011 The Author(s). Licensee IntechOpen. This chapter is distributed under the terms of the Creative Commons Attribution-NonCommercialShareAlike-3.0 License, which permits use, distribution and reproduction for non-commercial purposes, provided the original is properly cited and derivative works building on this content are distributed under the same license. 